\title{
Troubles ventilatoires et obésité Introduction
}

\section{J.-P. Bastard $\cdot$ M. Pigeyre $\cdot$ J. Dargent}

(C) Lavoisier SAS 2015

Chers lecteurs d'Obésité, ce numéro de septembre vous présente un dossier spécial sur les troubles ventilatoires, compliquant l'obésité, un vaste sujet de la pratique clinique quotidienne.

Comme vous pourrez le constater, nous avons eu la chance de réunir dans ce dossier des articles de spécialistes du domaine, illustré en préambule par un éditorial rédigé par le Pr O. Ziegler.

Nous avons associé dans ce numéro l'article du Dr R. Fuzier qui est pour nous complémentaire de l'article de l'équipe du Pr S. Jaber, puisqu'il traite de l'aspect locorégional de l'anesthésie chez le sujet obèse.

Sont présentés également dans ce numéro les résumés des présentations lors des Journées Benjamin Delessert 2015.

Enfin, nous retrouverons la revue de presse présentée par L. Genser et C. Barrat.

Nous espérons que vous apprécierez la lecture de ce numéro d'Obésité et bonne rentrée à tous ! 